\title{
Argumentation et interaction dans les brochures du Conseil fédéral suisse sur les votations populaires
}

Argumentation and Interaction in the Brochures of the Swiss Federal Council about the Popular Elections

Marc Bonhomme

\section{(2) OpenEdition Journals}

Édition électronique

URL : http://journals.openedition.org/aad/1454

DOI : 10.4000/aad.1454

ISSN : 1565-8961

Éditeur

Université de Tel-Aviv

\section{Référence électronique}

Marc Bonhomme, «Argumentation et interaction dans les brochures du Conseil fédéral suisse sur les votations populaires », Argumentation et Analyse du Discours [En ligne], 10 | 2013, mis en ligne le 10 avril 2013, consulté le 23 septembre 2019. URL : http://journals.openedition.org/aad/1454; DOI : $10.4000 /$ aad. 1454

Ce document a été généré automatiquement le 23 septembre 2019.

\section{cc)}

Argumentation \& analyse du discours est mis à disposition selon les termes de la licence Creative Commons Attribution - Pas d'Utilisation Commerciale - Pas de Modification 4.0 International. 


\section{Argumentation et interaction dans les brochures du Conseil fédéral suisse sur les votations populaires}

Argumentation and Interaction in the Brochures of the Swiss Federal Council about the Popular Elections

Marc Bonhomme

\section{Introduction}

1 En Suisse ont lieu plusieurs fois par an des scrutins populaires - ou des " votations $»^{1}-$ qui constituent le noyau de la démocratie directe helvétique et à l'occasion desquels les citoyens sont appelés à se prononcer sur des sujets touchant la politique fédérale, cantonale ou communale. Lors des votations engageant le pays en entier, après l'approbation du Parlement (appelé aussi Assemblée fédérale) sur leur validité, le Conseil fédéral ${ }^{2}$ envoie à chaque citoyen des brochures présentant les sujets soumis au suffrage du peuple. En raison du crédit dont bénéficient les instances fédérales auprès de la population, ces brochures établissent une communication politique primordiale pour l'orientation du scrutin. Entre 2001 et 2010, période qui va nous retenir, ces brochures ont concerné environ 80 votations. Ces dernières ont porté sur la politique suisse nationale ou internationale, de même que sur des questions économiques et sociales. Si ces documents s'inscrivent formellement dans les contraintes du genre "brochure ", leur contenu est composite, puisqu'ils doivent intégrer, outre le texte soumis à la votation, les explications du Conseil fédéral, les arguments avancés par les instigateurs du scrutin et les conseils de vote des autorités fédérales ${ }^{4}$. En somme, ces brochures répondent globalement à deux visées: exposer l'objet du vote et recommander la position gouvernementale.

2 L'objectif de notre article est d'étudier le fonctionnement argumentatif singulier de ce genre de texte politique, en nous focalisant sur la liaison étroite entre argumentation et interaction qu'il offre ${ }^{5}$. Pour ce faire, nous adoptons le cadre théorique des modèles dits 
«dialogaux» de l'argumentation, développés depuis quelque temps par Hamblin (1970), van Eemeren et Grootendorst (1996) ou Plantin (2005), selon lesquels les conduites argumentatives dépendent de leur contexte et des interactions en jeu dans ce dernier. De fait lors des votations, non seulement les brochures qui nous intéressent prennent place dans toute une chaîne de paroles, composée de discours qui se répètent ou qui se réfutent à travers les médias les plus divers (presse, radio, télévision...) et de la part des instances énonciatives les plus variées: hommes politiques, journalistes, simples citoyens... ${ }^{6}$ Mais elles déploient une argumentation doublement interactive. D'un côté, elles comportent une importante dimension interdiscursive, fondée sur la réaction à des énoncés antérieurement produits. Elles renferment en effet un patchwork de discours rapportés relatifs à l'objet du vote, qu'il s'agisse de déclarations politiques, de textes législatifs ou d'extraits des initiatives populaires sur lesquelles les autorités fédérales se prononcent. D'un autre côté, ces brochures contiennent des traces plus ou moins marquées d'une activité interlocutive, de nature prospective, avec les citoyens auxquels elles s'adressent ${ }^{7}$. Par ailleurs, ces deux modalités interactives qui conditionnent l'argumentativité de telles brochures peuvent être plus ou moins ouvertes sur le plan communicationnel, tout en oscillant sur le plan politique entre des convergences et des divergences d'opinions avec les positions qu'elles rapportent.

3 Nous nous proposons plus précisément de montrer comment ces modalités interactives, qu'elles soient interdiscursives ou interlocutives, fonctionnent à travers leur gradualité, en corrélation avec le jeu stratégique Explication-Recommandation à l'œuvre dans les brochures du Conseil fédéral. En particulier, à partir de cinq brochures représentatives, nous voudrions mettre en évidence les fluctuations de ce fonctionnement selon les situations des votations, qu'elles consistent en des modifications d'articles constitutionnels ou en des soumissions d'initiatives populaires ${ }^{8}$ que les autorités fédérales peuvent soutenir ou rejeter.

\section{Proposition de modification de la législation en bioéthique}

4 La première brochure que nous retenons concerne un projet de référendum constitutionnel destiné à remanier un article de loi. Intitulé «Recherche sur l'être humain » et soumis au peuple le 7 mars 2010, cet objet de votation touche un problème de société auquel le public est largement sensibilisé en raison de son actualité. Ce type de brochure présente une interaction en demi-teinte, comme le révèlent ses composantes didactiques et argumentatives.

\subsection{Didactisme et activité interlocutive en creux}

5 À l'instar des autres brochures du Conseil fédéral, celle évaluant la recherche sur l'être humain est foncièrement didactique, ce qui implique une interlocution constitutive avec le public qu'elle cible. Cette interlocution est asymétrique, car elle repose sur la communication d'un savoir, à travers laquelle le Conseil fédéral se trouve en position haute, d'un point de vue cognitif et illocutoire, par rapport au peuple suisse qu'il veut informer. En même temps, cette transmission d'un savoir se modèle sur son public en fonction de l'image que le Conseil fédéral s'en fait, ce qui est le propre de tout discours de vulgarisation. Mais une telle proaction interlocutive est ici en creux, le destinataire 
n'étant pas marqué explicitement dans le discours. Elle s'effectue essentiellement par l'injection de facilitateurs de lecture à trois niveaux du texte.

D'abord, quand on lit le titre de la brochure, on découvre la mention «Explications du Conseil fédéral ». Instaurant un cadrage assertif (Vanderveken 1988), ce titre engage un pacte de lecture pédagogique avec le lecteur-citoyen, même si ce pacte est ambigu, comme on le vérifiera.

7 De plus, au niveau de la composition rhétorique, on observe d'emblée deux phénomènes. D'une part, cette brochure est subdivisée en sections facilement identifiables, avec chaque fois un intitulé clair, l'ensemble proposant une réduction didactique de la complexité du sujet traité : «L'essentiel en bref », «L'objet en détail », « Texte soumis au vote "... D'autre part, on remarque à droite des sous-titres résumant les parties développées, comme l'illustre la section «L'essentiel en bref »:

\begin{tabular}{|c|c|}
\hline \multicolumn{2}{|l|}{ L'essentiel en bref } \\
\hline $\begin{array}{l}\text { [...] La médecine demande des connaissances renouvelées pour } \\
\text { les soins et la prévention des maladies. Cela serait impossible } \\
\text { sans la recherche sur l'être humain. À titre d'exemple, un } \\
\text { nouveau médicament ne pourrait être mis sur le marché si on ne } \\
\text { l'avait au préalable testé sur l'être humain avec de bons résultats. }\end{array}$ & $\begin{array}{l}\text { Rôle central de la } \\
\text { recherche }\end{array}$ \\
\hline $\begin{array}{l}\text { En Suisse la situation juridique concernant la recherche sur l'être } \\
\text { humain est insatisfaisante. Au niveau fédéral, ce domaine n'est } \\
\text { que partiellement réglé. Au niveau cantonal, les prescriptions } \\
\text { sont soit inexistantes, soit très disparates. Or la recherche sur } \\
\text { l'être humain pose des problèmes éthiques, d'où la nécessité d'en } \\
\text { fixer le cadre à l'échelle nationale. }\end{array}$ & $\begin{array}{l}\text { Nécessité d'une } \\
\text { réglementation } \\
\text { harmonisée }\end{array}$ \\
\hline $\begin{array}{l}\text { Lors des discussions parlementaires, il était entendu qu'il fallait } \\
\text { créer une base constitutionnelle. Quant à savoir s'il fallait fixer } \\
\text { des principes dans ce domaine dès le niveau constitutionnel, } \\
\text { c'était sujet à controverse. La majorité s'est prononcée pour cette } \\
\text { option, considérant l'article constitutionnel présenté ici comme } \\
\text { un juste milieu qui permet de protéger l'être humain de toute } \\
\text { dérive sans pour autant freiner la recherche. }\end{array}$ & Le juste milieu \\
\hline $\begin{array}{l}\text { Le Conseil fédéral et le Parlement sont convaincus que la } \\
\text { recherche sur l'être humain est indispensable. Ils souhaitent lui } \\
\text { donner un cadre clair et contraignant au niveau constitutionnel. }\end{array}$ & $\begin{array}{l}\text { Position du } \\
\text { Conseil fédéral et } \\
\text { du Parlement }\end{array}$ \\
\hline
\end{tabular}

8 Ces sous-titres aiguillent une lecture verticale sur la page, qui double la lecture horizontale du texte, selon un quadrillage strict de l'information. Ce dispositif se traduit par une forte vi/lisibilité qui anticipe une prise de connaissance rapide de la brochure pour ses destinataires. Cette commodité de lecture induite est renforcée par le fait que toutes les brochures du Conseil fédéral suivent cette même disposition, avec quelques variantes. On peut ainsi parler d'un canevas compositionnel stéréotypé qui joue sur la compétence interdiscursive du public et qui devance ses attentes. 
On relève d'autres facilitateurs de lecture au niveau de l'écriture de ces brochures, ou de leur élocution rhétorique. Parmi eux figure la simplicité de la syntaxe, typiquement journalistique, avec des phrases généralement brèves destinées à la mémoire à court terme du public. Ces phrases sont solidement articulées ${ }^{9}$, majoritairement au présent et sous une forme active, comme l'attestent les deux premiers paragraphes de «L'essentiel en bref» ci-dessus. Le recours constant à l'exemplification (voir entre autres au premier paragraphe la mention de l'expérimentation préalable des nouveaux médicaments) focalise aussi l'attention du lecteur, en ce qu'il établit une connexion avec son vécu concret. Mais l'activité de lecture est surtout guidée par une pratique systématique de la reformulation qui trouve son ancrage dans le "Texte soumis au vote ». Celui-ci est ensuite repris sous une forme condensée dans " L'essentiel en bref », puis sous une forme expansée dans « L'objet en détail »:

\begin{tabular}{|c|c|c|}
\hline Texte soumis au vote & $\begin{array}{l}\text { L'essentiel en } \\
\text { bref }\end{array}$ & L'objet en détail \\
\hline 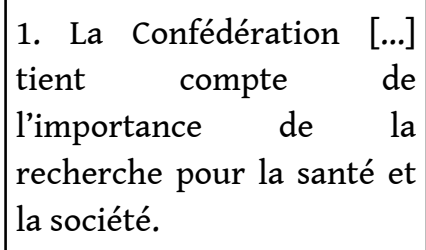 & $\begin{array}{l}\text { Rôle central de } \\
\text { la recherche }\end{array}$ & $\begin{array}{l}\text { L'article constitutionnel pose que la } \\
\text { recherche ne doit pas être inutilement } \\
\text { entravée et qu'il faut tenir compte de } \\
\text { son rôle central pour la santé et la } \\
\text { société. }\end{array}$ \\
\hline $\begin{array}{l}\text { 2. Un projet de recherche } \\
\text { ne peut être réalisé que si } \\
\text { la personne y participant } \\
\text { ou la personne désignée } \\
\text { par la loi a donné son } \\
\text { consentement éclairé. }\end{array}$ & \begin{tabular}{|lrr}
{$[\ldots .]$.} & un & cadre \\
clair & & et \\
contraignant \\
{$[\ldots]$} & &
\end{tabular} & $\begin{array}{l}\text { Une personne ne peut être amenée à } \\
\text { participer à un projet de recherche que } \\
\text { si elle a donné son consentement. Si } \\
\text { cette personne n'est pas en mesure de } \\
\text { prendre une décision d'une telle portée, } \\
\text { l'obligation au consentement repose } \\
\text { sur son représentant légal. }\end{array}$ \\
\hline $\begin{array}{l}\text { 3. Un projet de recherche } \\
\text { ne peut être réalisé sur des } \\
\text { personnes incapables de } \\
\text { discernement que si des } \\
\text { résultats équivalents ne } \\
\text { peuvent être obtenus sur } \\
\text { des personnes capables de } \\
\text { discernement. }\end{array}$ & \begin{tabular}{|lr}
{$[\ldots .$.$] \quad protéger$} \\
l'être & humain \\
de toute dérive \\
{$[\ldots .]$.}
\end{tabular} & $\begin{array}{l}\text { Les personnes qui ne sont pas en } \\
\text { mesure de consentir à leur } \\
\text { participation à un projet de recherche } \\
\text { (enfants en bas âge, personnes avec un } \\
\text { lourd handicap mental) doivent } \\
\text { bénéficier d'une protection accrue. [...] } \\
\text { Elles ne peuvent donc participer à un } \\
\text { projet de recherche que si les résultats } \\
\text { ne peuvent pas être obtenus avec des } \\
\text { adultes capables de discernement. }\end{array}$ \\
\hline
\end{tabular}

10 Le Conseil fédéral procède ainsi à un intense travail de réécriture, source d'un « dialogisme intralocutif » (Bres $2005: 53$ ), qui combine répétitions (comme « donn[er] son consentement » en 2), paraphrases (« importance de la recherche », " rôle central de la recherche » en 1) et commentaires (voir en 3 les précisions apportées par « L'objet en détail» sur les personnes incapables de discernement). Cette pratique métalinguistique de reprises et d'éclairages engendre dans la brochure une structure paradigmatique d'équivalences (au sens de Jakobson 1963) qui se superpose à sa 
progression syntagmatique. Le tout aboutit à un texte politique lourdement didactique, dont les modalités de lecture sont déjà balisées, ce qui pré-oriente la réception de l'argumentation transmise.

\subsection{Une argumentation interactivement ambiguë}

11 En effet, dans la brochure «Recherche sur l'être humain », loin d'être simplement juxtaposés, didactisme et argumentation participent à une même volonté d'adhésion du public à son $\operatorname{propos}^{10}$. En mettant en relief des arguments jugés pertinents, les facilitateurs de lecture qu'on a dégagés contribuent à leur force de conviction. De surcroît, la rationalisation de la composition et de l'élocution assurée par ces facilitateurs de lecture contient en elle-même un potentiel de persuasion. Toutefois, sur cette base, l'argumentativité proprement dite de cette brochure n'est pas dépourvue d'ambiguïté, au moins sur deux plans.

12 La première ambiguïté tient aux objectifs illocutoires divergents de cette brochure. On a vu que, dans son titre général («Explications du Conseil fédéral »), elle affiche un objectif assertif: expliquer. Mais au niveau de son encadrement textuel, elle formule simultanément un objectif directif. Énoncé sur la page initiale : « Le Conseil fédéral et le Parlement vous recommandent d'accepter l'article constitutionnel concernant la recherche sur l'être humain ", cet autre objectif est repris en conclusion à la dernière page. Or d'après Vanderveken (1988: 185), «recommander, c'est conseiller en présupposant que ce qui est recommandé est bon en général ». De la sorte, on a affaire ici à une visée interlocutive qui implique non plus la liberté du citoyen comme avec " expliquer ", mais une contrainte à son égard. Celle-ci est d'autant plus accentuée qu'elle émane d'un prescripteur collectif (le Conseil fédéral et le Parlement ${ }^{11}$ ) représentant les plus hautes autorités de l'État suisse.

13 Une autre ambiguïté argumentative de cette brochure réside dans sa tension entre une certaine ouverture interdiscursive et une communication autocentrée prédominante, illustrée par «L'essentiel en bref ». L'objet soumis au vote, à savoir la recherche réglementée sur l'être humain, est un thème qui a alors suscité beaucoup de débats de société en Suisse, qu'ils soient politiques ou médiatiques ${ }^{12}$. Or ces débats, d'ordre délibératif, sont soit rapidement pris en considération avec des allusions aux « discussions parlementaires » et à la « controverse » qui s'en est suivie, soit canalisés dans la ligne argumentative de la brochure, puisqu'elle mentionne essentiellement la position de la «majorité » qu'elle représente, mais très peu celle de la minorité qui va dans un sens inverse. En somme, l'arrière-plan interdiscursif des différentes prises de position antérieures sur le sujet est passablement escamoté.

14 Par contre, cette brochure tend à développer une argumentation bouclée sur les positions des autorités fédérales ${ }^{13}$. Dans «L'essentiel en bref », celle-ci se manifeste par des déclarations de principe, du genre: «la médecine demande des connaissances renouvelées ", «la recherche sur l'être humain pose des problèmes éthiques » ou « la recherche sur l'être humain est indispensable ». Ces déclarations de principe, assertées comme évidentes, sont en fait des topoï collectivement partagés ${ }^{14}$ et actualisés dans de nombreuses productions institutionnelles ou scientifiques, mais leur caractère interdiscursif est gommé au profit de l'énonciation exclusive du Conseil fédéral. Elles fournissent en outre les pivots d'une argumentation rationnelle, à la mécanique autosuffisante, qui part des besoins de la recherche, en passant par les lacunes de la 
législation en vigueur, pour en arriver à l'obligation de modifier celle-ci. Cet enchaînement logique est étayé par des marquages négatifs ("cela serait impossible ») et péremptoires (" nécessité », "convaincus», "contraignant») qui ferment par avance toute contre-argumentation. Au total, on assiste à la mise en place d'un discours de proposition catégorique, fondé sur une rationalité éthique, qui est fortement autolocuté et qui paraît ne laisser guère le choix au citoyen. Ce faisant, le Conseil fédéral construit un ethos autoritaire de garant du bien public, mais aussi un ethos modéré, comme le révèle "le juste milieu ", typiquement helvétique, revendiqué par l'un des sous-titres de « L'essentiel en bref ».

\section{Prises de position du Conseil fédéral sur des initiatives populaires}

Avec la brochure "Recherche sur l'être humain ", le Conseil fédéral endosse finalement un rôle de proposant $t^{15}$, à travers une interaction encore en retrait. Cependant, la plupart de ses brochures constituent des prises de position réagissant à des initiatives populaires, ce qui entraîne une montée au premier plan de leur dimension interactive.

\subsection{Brochure de soutien d'une initiative populaire. Le cas de l'adhésion à l'ONU}

Le Conseil fédéral se prononce assez rarement en faveur d'une initiative populaire et celle pour une adhésion de la Suisse à l'ONU, soumise au vote le 3 mars 2002, en est un bel exemple. Tout en s'avérant capitale pour la politique étrangère helvétique, cette question était éminemment sensible à l'époque. Après l'échec de la votation sur le même sujet en 1986, malgré le soutien des milieux politiques et économiques, la population restait profondément divisée à son égard. Confrontée à ce terrain miné, la brochure du Conseil fédéral met en place un double dispositif interactif. Dans une énonciation convergente, elle renchérit sur les arguments du comité d'initiative ${ }^{16}$ pour une adhésion à l'ONU. Mais conjointement, dans un mouvement de désamorçage, elle fait tout pour contrer par avance le courant populaire hostile à l'ONU de façon à rendre inattaquable le projet présenté.

\subsubsection{Un important bétonnage pédagogique interlocutif}

On retrouve dans cette brochure de soutien à l'initiative en faveur de l'ONU les éléments pédagogiques relevés dans la brochure précédente : décomposition en parties claires («L'essentiel en bref», «Avis du Conseil fédéral »...), reformulations réglées entre ces parties... Mais cette brochure se singularise par l'ajout de plusieurs rubriques explicatives qui tranchent avec la structure canonique des autres brochures. Ainsi, deux pages entières sont consacrées aux succès politiques de l'ONU et une page expose les réalisations de cet organisme dans les domaines économiques et sociaux. Un tel luxe d'explications, très orientées argumentativement, vise - sur le plan interlocutif - à donner le maximum d'informations positives à l'électeur hésitant ou sceptique et à provoquer son ralliement à la cause de l'ONU. L'électeur est du reste directement interpellé à l'occasion, en communauté avec l'énonciateur politique: «Ce que fait l'ONU nous regarde » (p. 6). 
Par ailleurs, la fin de cette brochure renferme un bulletin de commande de la charte de l'ONU pour des informations complémentaires. L'insistance est mise sur la facilité d'acquisition de celle-ci : voie postale, appel téléphonique, téléchargement sur Internet. Quant à la rétroaction du lecteur-citoyen, elle est stimulée par une incitation lui garantissant une absence de dépense ("Vous pouvez vous procurer la charte gratuitement») et par une anticipation de son acte de commande : "Je commande ... exemplaire(s) de la Charte de l'ONU ». Bref, tout est fait pour pousser le lecteur à conforter lui-même son opinion et à se décider en pleine connaissance.

\subsubsection{Une surenchère argumentative}

19 Ce bétonnage en vue d'une information maximale du lecteur se prolonge par une surenchère dans l'argumentation, laquelle fonctionne selon trois perspectives.

En premier lieu, sur le mode d'une énonciation interdiscursive convergente, le Conseil fédéral reprend avec insistance les arguments du comité d'initiative favorables à une adhésion à l'ONU. Synthétisée sur le tableau ci-après, cette activité d'étayage en écho les amplifie et leur confère un statut officiel, suite à leur prise en charge gouvernementale :

\begin{tabular}{|c|c|}
\hline "Arguments du comité d'initiative" & « Avis du Conseil fédéral » \\
\hline $\begin{array}{l}\text { - Mondialisation: } \\
\text { «Le cœur de l'économie bat désormais au } \\
\text { rythme de la mondialisation. Les } \\
\text { catastrophes écologiques et le terrorisme } \\
\text { ne connaissent plus de frontières.» }\end{array}$ & $\begin{array}{l}\text { « À l'heure où la mondialisation } \\
\text { s'intensifie, des défis dépassant le cadre } \\
\text { national doivent être relevés dans tous } \\
\text { les secteurs de la vie quotidienne." }\end{array}$ \\
\hline $\begin{array}{l}\text { - Identité de vues: } \\
\text { «Ses [l'ONU] objectifs et ses moyens } \\
\text { d'action sont partagés par la plupart des } \\
\text { Suisses.» }\end{array}$ & $\begin{array}{l}\text { «L'ONU et la Suisse sont à l'unisson et } \\
\text { poursuivent les mêmes objectifs.» }\end{array}$ \\
\hline $\begin{array}{l}\text { - Collaboration déjà existante: } \\
\text { « Nous participons depuis longtemps aux } \\
\text { programmes de l'ONU en faveur des plus } \\
\text { défavorisés.» }\end{array}$ & $\begin{array}{l}\text { «La Suisse et l'ONU travaillent ensemble } \\
\text { depuis de très nombreuses années. » }\end{array}$ \\
\hline $\begin{array}{l}\text { - Non adhésion problématique: } \\
\text { «Aucun des problèmes que nous } \\
\text { connaissons aujourd'hui ne pourra être } \\
\text { résolu facilement si nous restons à l'écart } \\
\text { de l'ONU.» }\end{array}$ & $\begin{array}{l}\text { «Les possibilités dont [notre pays] } \\
\text { dispose pour faire valoir ses intérêts sont } \\
\text { limitées, car il ne peut pas participer aux } \\
\text { votes.» }\end{array}$ \\
\hline
\end{tabular}

21 En deuxième lieu, sur le mode d'une énonciation interdiscursive divergente, dans une rubrique intitulée «Les opposants ont dit» (p.10), la brochure rapporte quelques arguments à l'encontre d'une adhésion de la Suisse à l'ONU émanant des débats au Parlement. Mais ces arguments hostiles se voient triplement minorés. D'abord, il est 
précisé qu'ils ne représentent pas l'avis du peuple: "Aucun comité d'initiative ne pouvant présenter le point de vue des adversaires du projet, vous trouverez [...]». Ensuite, ces arguments hostiles pèchent par leur absence de référence. On ignore ainsi l'identité des opposants à l'ONU qui apparaissent comme des voix anonymes face aux voix assumées du comité d'initiative et du Conseil fédéral. Enfin, ces arguments hostiles sont mentionnés à travers un cumul désordonné qui leur enlève toute cohérence argumentative. Par exemple, l'argument de la souveraineté de la Suisse hors de l'ONU est formulé dans les premières citations, puis il laisse la place à une juxtaposition d'arguments hétéroclites pour réapparaitre dans la dernière citation. Ce cheminement erratique contraste avec le plaidoyer méthodique du Conseil fédéral en faveur de l'ONU.

Surtout, cette brochure développe, à un troisième niveau, une batterie d'arguments énoncés par le Conseil fédéral afin de convaincre, sur le mode interlocutif, les électeurs les plus réticents. Diffus dans toute la brochure, y compris dans ses séquences didactiques, ces arguments préconisant une adhésion à l'ONU sont de deux ordres. D'un côté, la brochure exploite l'argument du bénéfice ${ }^{17}$, avec sa double configuration :

23 a) Les avantages d'une adhésion à l'ONU sont optimaux, en ce qu'ils apportent à la Suisse un gain par rapport à la situation actuelle: «Devenir membre permettra à la Suisse d'exercer les mêmes droits que les autres États » (p. 6) - «En adhérant à l'ONU, la Suisse pourra mieux défendre ses intérêts et promouvoir ses valeur » (p. 12).

b) Inversement, une adhésion à l'ONU n'occasionnera pas de perte pour la Suisse. Politiquement, elle conservera sa neutralité, ce qui constitue un argument capital pour beaucoup d'électeurs: "En devenant membre de l'ONU, la Suisse restera neutre » (p. 13). Financièrement, l'adhésion à l'ONU ne grèvera pas le budget de l'État : « Le coût de l'adhésion est raisonnable et tout à fait supportable pour les finances fédérales » (p. 13). Militairement, cette adhésion ne nécessitera pas de contrepartie : «[La Suisse] ne sera pas contrainte de participer aux opérations militaires de l'ONU » (p. 5).

D'un autre côté, la brochure recourt à l'argumentation par les valeurs communes à la Suisse et à l'ONU, qu'elles soient humanitaires ou environnementales. L'extrait suivant est symptomatique à cet égard: "L'ONU protège les droits de l'homme, apporte son secours en cas de catastrophe, lutte contre la pauvreté et est une des locomotives de la protection de l'environnement. [...] Ces domaines d'action sont également prioritaires pour la Suisse » (p. 12). Une telle mise en avant d'arguments idéalistes vise à entraîner proactivement la communion (dans l'acception de Perelman et Olbrechts-Tyteca 1988 ${ }^{18}$ ) du lecteur-citoyen à ces valeurs partagées par tous.

Dans la même stratégie d'une proaction interlocutive sur le lecteur, la brochure déploie des arguments plus spécifiques. Elle utilise l'argumentation par les chiffres pour souligner l'importance des institutions et des organismes approuvant une adhésion à l'ONU ${ }^{19}$, à l'exemple de cet encart :

Une adhésion largement soutenue

Les cantons, les partis, les milieux économiques et de nombreuses associations sont très largement favorables à l'adhésion de la Suisse à l'ONU. La consultation menée en été 2000 a donné les résultats suivants : tous les gouvernements cantonaux, à l'exception d'Appenzell Rhodes-Intérieures, soutiennent l'adhésion. Sur les 8 partis politiques représentés au Parlement, 6 sont favorables à l'adhésion et 2 s'y opposent. 6 sur 7 associations faîtières de l'économie défendent l'adhésion [...]. Sur les quelque 50 organisations non gouvernementales consultées, plus de 40 se sont exprimées pour l'adhésion. 

rapide chez le lecteur. Entre autres, à la page $5:$ «Il est grand temps que la Suisse adhère à l'ONU, car elle n'a aucune raison de rester à l'écart ». À cela s'ajoute un usage abondant de l'argument de proximité, destiné à désamorcer chez le lecteur l'idée selon laquelle l'ONU est un organisme distant et froid. Ainsi apparaissent en bonne place le fait que Genève est le deuxième siège de l'ONU (p. 13), ou le fait que, par l'intermédiaire de l'UNESCO, « [elle] protège des monuments historiques, tels que [...] le couvent de St Gall, la vieille ville de Berne et les châteaux de Bellinzona » (p. 8). En somme, l'ONU est une « famille » (p. 8) dont le citoyen suisse ne saurait s'exclure.

l'argument sans doute le plus déterminant pour persuader proactivement le lecteur incertain est la figuration à la page 9 d'un fac-similé de la demande d'adhésion de la Suisse à l'ONU adressée à Kofi Annan par le Conseil fédéral :

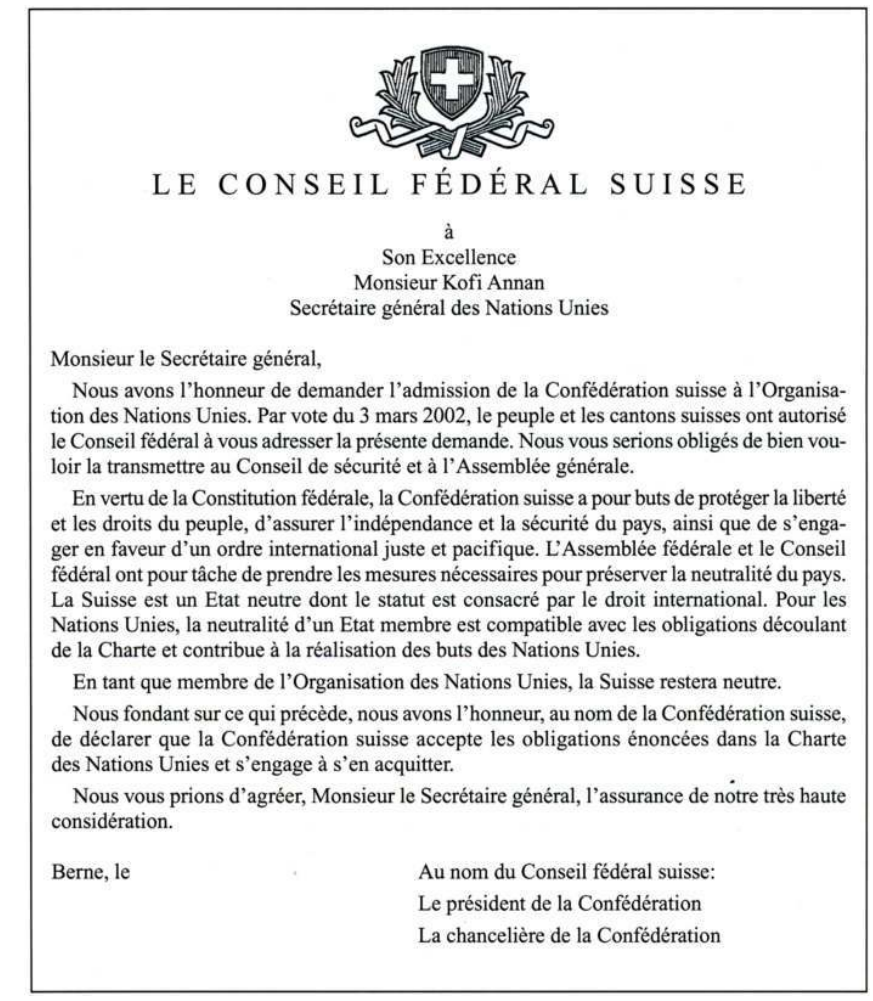

29 Cette reproduction constitue un argument proleptique qui pré-oriente le choix de l'électeur dans un sens se voulant décisif et qui anticipe déjà le succès de la votation ${ }^{20}$.

\subsection{Brochures de rejet d'une initiative populaire}

Le plus souvent, les autorités fédérales se prononcent contre les initiatives proposées aux votations populaires. En résulte une interaction plus nettement antagonique qui varie selon les formes que prend ce rejet.

\subsubsection{Rejet sur la base d'un accord de principe : l'exemple des aliments sans manipulations génétiques}

31 Soumise au vote le 27 novembre 2005, l'initiative populaire "Pour des aliments produits sans manipulations génétiques " porte sur une thématique écologique qui bénéficie d'un large consensus en Suisse. Pourtant, les autorités fédérales l'ont rejetée, 
certes à une faible majorité. Un tel rejet contre la doxa prédominante implique, dans la brochure du Conseil fédéral, la mise en place d'une argumentation nuancée dont il convient d'examiner les principaux aspects.

Les « Arguments du comité d'initiative » rapportés dans la brochure se remarquent par leur interlocution très explicite avec la population appelée à voter. D'une part, la section mentionnant ces arguments est ostensiblement encadrée par un appel au vote initial: "Votez oui à l'initiative sans OGM ", appel au vote qui se voit repris dans sa conclusion: "Ce sont là de bonnes raisons de voter oui à l'initiative sans OGM ». Ensuite cette section est parsemée de déictiques personnels associant dans une visée énonciative commune les auteurs de l'initiative et la population sollicitée: «Cela incitera nos partenaires commerciaux à rester aussi sans OGM » - « Nous préservons la biodiversité ». En outre, cette section unit dans une même condamnation des OGM l'ensemble des acteurs économiques: paysans, défenseurs de l'environnement et consommateurs.

Face à ce positionnement argumentatif tranché, comment réagit le Conseil fédéral dans sa brochure ? Il adopte une énonciation interdiscursive concordante pour le fond de la question, car il reconnaît lui aussi le problème des OGM et la nécessité de leur réglementation, en rappelant la loi sur ce même sujet dans "L'essentiel en bref " (p.5): «La loi de 2004 sur le génie génétique [...] interdit l'utilisation d'animaux génétiquement modifiés dans l'agriculture et subordonne l'octroi de l'autorisation d'utiliser une plante génétiquement modifiée à toute une série d'essais ».

Mais en même temps, la brochure du Conseil fédéral recourt à une énonciation interdiscursive discordante avec le comité d'initiative pour la forme ou la pertinence de ses propositions, en mettant en avant leur inutilité. Ce faisant, le Conseil fédéral inscrit son argumentation au cœur du registre délibératif, puisque l'inutile avec son contraire, l'utile, en constitue l'un des grands topoi ${ }^{21}$. Le topos de l'inutile est réitéré à maintes reprises dans la brochure, en particulier pour souligner la superfluité de l'initiative avec la législation existante : «L'initiative ne protège pas mieux l'homme, les animaux et l'environnement que la loi sur le génie génétique. Cette loi [...] répond donc déjà largement aux exigences de l'initiative »(p.10). L'inutilité de l'initiative est également démontrée au moyen d'un tableau comparatif (p. 8):

\begin{tabular}{|l|l|l|l|}
\hline & Initiative & $\begin{array}{l}\text { Réglementation en } \\
\text { vigueur (loi sur le génie } \\
\text { génétique) }\end{array}$ & $\begin{array}{l}\text { Situation actuelle } \\
\text { (septembre 2005) }\end{array}$ \\
\hline $\begin{array}{l}\text { Culture de plantes } \\
\text { génétiquement modifiées }\end{array}$ & $\begin{array}{l}\text { Interdite } \\
\text { pendant cinq } \\
\text { ans }\end{array}$ & $\begin{array}{l}\text { Possible après une } \\
\text { procédure de contrôle et } \\
\text { d'essais très stricte }\end{array}$ & $\begin{array}{l}\text { Aucune culture : aucune } \\
\text { demande déposée }\end{array}$ \\
\hline $\begin{array}{l}\text { Utilisation d'animaux de } \\
\text { rente génétiquement } \\
\text { modifiés dans l'agriculture }\end{array}$ & $\begin{array}{l}\text { pendant cinq } \\
\text { ans }\end{array}$ & Interdite & Aucune utilisation \\
\hline $\begin{array}{l}\text { Importation de denrées } \\
\text { alimentaires } \\
\text { génétiquement modifiées }\end{array}$ & $\varnothing$ & $\begin{array}{l}\text { Possible sur autorisation; ; } \\
\text { désignation obligatoire }\end{array}$ & $\begin{array}{l}\text { Peu d'importations de } \\
\text { denrées alimentaires } \\
\text { contenant des OGM }\end{array}$ \\
\hline
\end{tabular}




\begin{tabular}{|l|l|l|l|}
\hline $\begin{array}{l}\text { Importation d'aliments } \\
\text { génétiquement modifiés } \\
\text { pour l'alimentation du } \\
\text { bétail }\end{array}$ & $\varnothing$ & $\begin{array}{l}\text { Possible sur autorisation ; } \\
\text { désignation obligatoire }\end{array}$ & $\begin{array}{l}\text { Peu d'importations } \\
\text { d'aliments pour animaux } \\
\text { concernant des OGM }\end{array}$ \\
\hline & & & \\
\hline
\end{tabular}

L'intérêt de ce tableau est qu'il se présente comme pédagogique par sa schématisation, mais qu'il est implicitement argumentatif, avec une gradation dans le raisonnement. $\mathrm{Au}$ début, il laisse entendre que l'initiative est redondante par rapport à la situation actuelle. En effet, elle préconise l'interdiction de la culture de plantes et de l'utilisation d'animaux génétiquement modifiés, alors que ces pratiques sont inexistantes en Suisse. Par la suite, ce tableau suggère que la situation actuelle va plus loin, en matière de restriction sanitaire, que l'initiative qui ne prévoit rien (voir les cases vides) à propos de l'importation d'aliments génétiquement modifiés. D'où l'inférence que le lecteur est invité à tirer : l'initiative est non seulement inutile, mais lacunaire. Au bout du compte, la stratégie du Conseil fédéral consiste à couper l'herbe sous les pieds des auteurs de l'initiative et à nier le bien-fondé de leur démarche.

\subsubsection{Rejet avec contre-projet sur les étrangers criminels}

Une autre variante de rejet nuancé de la part des autorités fédérales concerne le cas où ce rejet s'accompagne d'un contre-projet de ces dernières ${ }^{22}$. Le but de ce contre-projet est de rester dans la même ligne que l'initiative proposée, tout en réorientant ses aspects jugés inacceptables. On a donc une stratégie de rebond sur une initiative populaire et de correction de celle-ci, avec un désaccord qui porte non plus sur la procédure comme précédemment, mais sur le fond de la question.

Cette stratégie est typique lors de l'initiative populaire de 2010 «Pour le renvoi des étrangers criminels». Bien qu'elles soient d'accord avec l'objectif global de cette initiative, à savoir la reconduite des étrangers coupables d'un délit hors des frontières helvétiques, les autorités fédérales nuancent très sérieusement les conditions d'application de cet objectif dans leur contre-projet intitulé «L'expulsion et le renvoi des criminels étrangers dans le respect de la Constitution ». Cette situation donne lieu, dans la mise en texte de la brochure du Conseil fédéral, à une argumentation interdiscursive croisée dont nous ne retenons que les traits essentiels.

L'initiative populaire et le contre-projet des autorités fédérales s'appuient sur un même principe clairement explicité dans la brochure: "L'initiative et le contre-projet proposent des règles contraignantes pour le retrait du droit de séjour des étrangers criminels » (p. 14). Toutefois, aussi bien dans «L'essentiel en bref» que dans «L'objet en détail », la brochure fait état des différences importantes entre les deux textes soumis au peuple. En gros, alors que l'initiative populaire envisage des mesures uniquement répressives et cela d'une façon mécanique à partir d'une liste ciblée de délits, le contre-projet conditionne les mesures répressives à la nature des délits perpétrés, sans en établir une liste préalable. Il prévoit en outre, grâce à une politique d'intégration renforcée, des mesures de prévention destinées à limiter les délits commis par la population étrangère. 
Sur cette base factuelle, la brochure relate les débats croisés à propos des deux positions en jeu qu'on peut ainsi synthétiser :

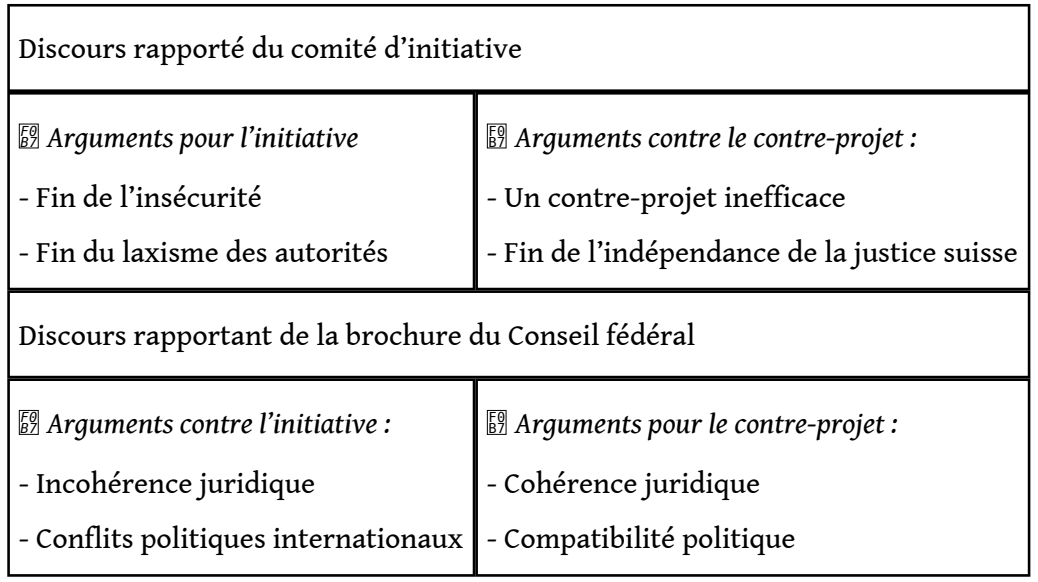

Plus précisément, dans la rubrique « Les arguments du comité d'initiative » (p. 12), la brochure mentionne les justifications de celui-ci en faveur de l'initiative ${ }^{23}$ et ses objections à l'encontre du contre-projet gouvernemental dont l'approche est estimée «totalement inopportune». Dans les deux cas, les arguments du comité d'initiative revêtent une tonalité dramatisante, avec des affirmations comme: "Les personnes âgées ne sont pas les seules à ne plus oser sortir le soir : de nombreux jeunes ont affaire tous les jours à des insultes et des bagarres». Cette tonalité dramatisante se double d'une tonalité nationaliste quand le comité d'initiative reproche au contre-projet de viser « à inscrire le droit international dans la Constitution et à le placer au-dessus du droit suisse ", avant d'ajouter : "Des avocats et des juges pourraient ainsi continuer à empêcher le renvoi de grands criminels». Pour sa part, dans la rubrique "Les arguments du Conseil fédéral » (pp. 14-15), ce dernier raisonne principalement à partir des topoï du désordre et de l'ordre ${ }^{24}$. Il met l'accent sur les désordres juridiques provoqués par l'initiative populaire qui transgresse la règle de justice :

C'est ainsi qu'un jeune étranger ayant grandi en Suisse devrait être automatiquement renvoyé pour un vol insignifiant [...]. Un adulte, par contre, [...] échapperait au renvoi automatique, même après avoir commis une escroquerie de grande envergure, parce que l'escroquerie ne fait pas partie des infractions mentionnées dans le texte de l'initiative.

41 Le Conseil fédéral signale pareillement les désordres politiques induits par cette initiative, vu que « d'importants accords de droit international ne pourraient plus être respectés, par exemple l'accord sur la libre circulation avec l'UE». En revanche, la brochure insiste sur l'ordre attaché au contre-projet gouvernemental, que cet ordre soit juridique, avec une sanction adaptée au délit commis («Le contre-projet garantit une pratique équitable des autorités en matière de renvoi des étrangers coupables de graves infractions »), ou qu'il soit politique, dans la mesure où le contre-projet « est compatible avec [...] les principes de la Constitution et [qu'] il respecte les engagements pris par la Suisse à l'échelon international ». Bref, alors que l'initiative populaire paraît suivre la passion du moment, le contre-projet gouvernemental s'inscrit dans la raison, avec l'ethos de discernement et de responsabilité que cela suppose.

Par-delà ces prises de position argumentatives croisées, la brochure sur le renvoi des étrangers criminels utilise des modalités énonciatives différenciées quand elle expose 
les arguments de l'initiative populaire et ceux du Conseil fédéral. Les premiers sont souvent rapportés par des verbes énonciativement faibles: "L'initiative prévoit de $[\ldots .] ».($ p. $6,7,11)$ - «L'initiative estime que $[. .] ».($ p. 8) - «L'initiative ne propose rien [...]» (p.11)... Par contre, le Conseil fédéral emploie fréquemment des verbes énonciativement forts, entre autres des performatifs, pour donner plus de poids à la présentation du contre-projet : « Le contre-projet exige que [...]» (p. 10) - «Le Conseil fédéral soutient le contre-projet» (p.14) - «Le Conseil fédéral préconise le contreprojet » (p. 15) - «Le contre-projet garantit [...]» (p. 15)... Une telle surénonciation ${ }^{25}$, combinée avec les arguments d'ordre et de justice qu'on a vus, vise évidemment, dans une optique interlocutive, à faire pencher les électeurs en faveur du contre-projet.

\subsubsection{Rejet polémique à propos des abus dans le droit d'asile}

43 Il arrive enfin que le désaccord soit total entre les auteurs d'une initiative populaire et les autorités fédérales. En résultent des brochures véritablement conflictuelles, reposant sur une interaction polémique entre les points de vue en confrontation. Si l'on continue avec le sujet sensible de l'immigration, cette interaction polémique est révélatrice dans une brochure de 2002 réagissant à une initiative déposée par l'UDC, un parti suisse très marqué à droite et désireux de durcir la politique contre les réfugiés. Intitulée «Contre les abus dans le droit d'asile » et soumise en votation le 24 novembre 2002, cette initiative veut refouler les demandeurs d'asile dans " l'État tiers réputé sûr " (p.5) à travers lequel ils sont arrivés en Suisse. Par exemple, si un réfugié venait en Suisse en passant par la France ou l'Allemagne, pays démocratiques où sa vie ne serait pas en danger, il se verrait automatiquement renvoyé vers ces pays. Le but affiché du comité d'initiative est de lutter contre les faux réfugiés qui abusent du droit d'asile. Comme l'illustre la section de la brochure «Avis du Conseil fédéral» (p. 8-9) prenant position sur cette initiative, la réaction de celui-ci est très violente, en ce qu'elle en conteste à la fois le contenu et les fondements éthiques.

Endossant un rôle de discours-agent, la brochure développe une communication agonale $^{26}$ qui effectue une attaque en règle de l'argumentation du comité d'initiative, traitée comme discours-patient ${ }^{27}$ selon trois procédés interdiscursifs. Pour commencer, elle opère une disjonction énonciative entre les deux camps en présence. Au « leurs revendications " des auteurs de l'initiative, mentionnées avec un déterminant de distanciation, elle oppose le « notre projet de révision » du Conseil fédéral, recourant à un déterminant possessif qui marque une personnalisation et une solidarité collective dans l'action. Il est à noter que le Conseil fédéral use habituellement de la troisième personne pour parler de lui-même et que cette montée au créneau du « nous » est rare dans ses productions. Par ailleurs, la brochure déploie une stratégie de démasquage sur les propositions du comité d'initiative. Celle-ci privilégie la figure de l'enthymème pour dévoiler les buts inavoués de l'initiative, tels qu'ils apparaissent dans ce passage :

La revendication principale des auteurs de l'initiative - la non-entrée en matière sur les demandes des requérants ayant transité par un État réputé sûr- est irréalisable car $95 \%$ des requérants arrivent en Suisse par la voie terrestre, donc passent obligatoirement par un des pays limitrophes. Or tous les pays qui nous entourent sont des États tiers réputés sûrs, à qui ces requérants auraient pu demander l'asile. Ce que veulent donc les auteurs de l'initiative, c'est que dorénavant nous n'entrions plus en matière sur la quasi-totalité des demandes d'asile.

Ce raisonnement répond à la structure argumentative : 
Majeure: La revendication de l'initiative est irréalisable

Étayage: Car la plupart des requérants d'asile viennent en Suisse via des pays limitrophes.

Mineure: Or tous les pays limitrophes sont des États tiers réputés sûrs.

Conclusion: Donc l'initiative veut en fait restreindre drastiquement le traitement

des demandes d'asile.

46 travers l'interprétation du Conseil fédéral, une initiative contre le droit d'asile luimême, ce qui porte atteinte à l'un des piliers de la politique internationale suisse. Plus généralement, la brochure se livre à une réfutation point par point du discours proposant du comité d'initiative, résumé à la page 7, les arguments de ce dernier se voyant inversés par le discours opposant du Conseil fédéral. Jouant notamment sur les modalités véridictoires ${ }^{28}$, celui-ci transforme en "semblant de solution » le renvoi des immigrés dans un État tiers, qui est présenté comme «une solution» par le comité d'initiative. De même, quand le comité d'initiative affirme que ses propositions "amélioreront la situation existante», le Conseil fédéral réplique qu'elles «n'apporteront aucune amélioration». Ou encore, lorsque les auteurs de l'initiative soutiennent qu'elle "facilitera l'expulsion des faux réfugiés", le Conseil fédéral rétorque que « le renvoi ne pourra se faire et le requérant restera en Suisse ». Du reste, non seulement la brochure retourne les arguments de l'initiative, mais elle en explicite les incohérences qui conduisent à l'autophagie ${ }^{29}$. En particulier, ce qui est revendiqué comme un gain financier par le comité d'initiative ${ }^{30}$ est dénoncé comme un coût financier par le Conseil fédéral, avec ce commentaire: "C'est un non-sens! Cette initiative est contradictoire : d'un côté, elle dénonce le coût élevé de l'asile et de l'autre, elle provoque une nouvelle augmentation des frais d'assistance en interdisant à certaines personnes de travailler $»$.

Parallèlement, cette argumentation agonale s'accompagne d'une condamnation éthique très ferme du comité d'initiative, ce qui est inhabituel dans les brochures du Conseil fédéral dont le ton s'avère ordinairement réservé. Ainsi, en jugeant l'initiative " choquante », « déloyale » et " contraire à la tradition humanitaire de notre pays », en la considérant comme "une source d'injustice » et comme obligeant à «manquer à notre devoir de solidarité envers nos voisins ", le Conseil fédéral porte le débat sur le terrain axiologique, se donnant une posture de garant des valeurs altruistes liées à la Suisse. Par la même occasion, il s'en prend aux présupposés mensongers de l'initiative, comme l'atteste le début de son avis : «Les auteurs de l'initiative sous-entendent que nous pouvons renvoyer sans problème dans un État tiers réputé sûr le demandeur d'asile qui est arrivé en Suisse [...]. C'est une tromperie !».

\section{Conclusion}

Nous avons essayé de montrer comment, par-delà leur stéréotypie formelle, les brochures du Conseil fédéral lors des votations populaires suisses mettent en jeu une corrélation non seulement étroite, mais aussi très riche entre argumentation et interaction. Cette corrélation se traduit par une importante circulation des argumentaires, qu'elle soit interdiscursive, surtout vis-à-vis des initiatives populaires présentées, ou interlocutive par rapport aux citoyens appelés à voter. À partir des observations que nous avons faites, deux grandes tendances se dégagent :

Argumentation et Analyse du Discours, 10 | 2013 
a) Quand ces brochures expliquent et défendent une proposition de modification législative, elles offrent une interaction limitée, penchant vers l'argumentation autolocutée.

b) Par contre, quand elles commentent et évaluent des initiatives populaires, elles recèlent une forte activité interlocutive avec les lecteurs, du moment que l'issue de la votation n'est pas évidente. En même temps, elles comportent des stratégies interdiscursives variées, qui sont convergentes lorsqu'elles reprennent ou modulent les arguments des initiatives, mais qui apparaissent divergentes, voire polémiques, lorsqu'elles les réfutent.

Cette mise en circulation d'argumentaires diversifiés thématiquement, mais unifiés dans leur visée de persuasion des citoyens suisses s'effectue à travers la position énonciative doublement fluctuante du Conseil fédéral. Sur le plan institutionnel, il endosse tantôt le rôle de porte-parole du Parlement, tantôt celui de responsable de la politique gouvernementale. Envers le public auquel il s'adresse, il alterne entre des postures de commentateur et de prescripteur quant à l'objet des votations.

Plus largement et sans entrer dans les détails, car cela relève d'un autre type d'analyse, se pose à présent le problème de l'impact (persuasion effects) de ces brochures sur l'issue $\mathrm{du}$ vote. Cet impact pourrait être mesuré en comparant les recommandations du Conseil fédéral avec les résultats des votations examinées :

1) Proposition de modification de la législation: "Recherche sur l'être humain »

(2010)

- Conseil fédéral : Recommandation d'acceptation

- Votation : Acceptation (77, $2 \%)$

2) Initiative « Pour l'adhésion à l'ONU » (2002)

- Conseil fédéral : Recommandation d'acceptation

- Votation : Acceptation (54,6 \%)

3) Initiative « Pour des aliments produits sans manipulations génétiques » (2005)

- Conseil fédéral : Recommandation de rejet

- Votation : Acceptation (55,7\%)

4) Initiative « Pour le renvoi des étrangers criminels » (2010)

- Conseil fédéral : Recommandation de rejet

- Votation : Acceptation (52,3 \%)

+ Contre-projet de l'Assemblée fédérale

- Conseil fédéral : Recommandation d'acceptation

- Votation : Rejet (52,6\%)

5) Initiative « Contre les abus dans le droit d'asile » (2002)

- Conseil fédéral : Recommandation de rejet

- Votation : Rejet $(50,1 \%)$

51 On constate que, sur les cinq objets que nous avons retenus, le peuple a voté trois fois dans le sens des recommandations du Conseil fédéral, mais qu'il ne l'a pas suivi deux fois. Notre tableau statistique n'est donc pas très probant. En fait, en dépit de son autorité, le Conseil fédéral - avec ses brochures - n'est qu'un maillon dans les campagnes électorales, durant lesquelles les partis politiques, les associations professionnelles et les différents lobbies jouent aussi un rôle influent. De surcroît, dans un État décentralisé comme la Suisse, le Conseil fédéral est loin de dominer tous les paramètres, spécialement régionaux ou sociologiques, qui interviennent lors des votations. Mais c'est là un autre débat. 


\section{BIBLIOGRAPHIE}

Aristote. 1967. Rhétorique (Paris : Les Belles Lettres)

Bres, Jacques. 2005. «Savoir de quoi on parle : dialogue, dialogal, dialogique ; dialogisme, polyphonie... », Bres, Jacques et al. (éds). Dialogisme et polyphonie (Bruxelles : De Boeck-Duculot), 47-61

Desrosières, Alain. 2000 [1993]. La politique des grands nombres (Paris : La Découverte)

Greimas, Algirdas Julien \& Joseph Courtés. 1979. Sémiotique. Dictionnaire raisonné de la théorie du langage (Paris : Hachette)

Grize, Jean-Blaise. 1981. « L'argumentation : explication ou séduction », L'argumentation (Lyon : Presses Universitaires de Lyon), 29-40

Hamblin, Charles Leonard. 1970. Fallacies (London: Methuen)

Jacques, Francis. 1985. L'espace logique de l'interlocution (Paris : PUF)

Jakobson, Roman. 1963. Essais de linguistique générale (Paris : Minuit)

Maingueneau, Dominique. 1983. Sémantique de la polémique (Lausanne : L’Âge d'Homme)

Meyer, Michel. 2008. Principia Rhetorica (Paris : Fayard)

Moirand, Sophie. 2002. « Dialogisme », Charaudeau, Patrick \& Dominique Maingueneau (éds). Dictionnaire d'analyse du discours (Paris : Le Seuil), 175-178

Ogien, Albert. 2010. « La valeur sociale du chiffre. La quantification de l'action publique entre performance et démocratie ", Revue française de socio-économie 5, 19-40

Perelman, Chaïm \& Lucie Olbrechts-Tyteca. 1988 [1958]. Traité de l'argumentation (Bruxelles :

Éditions de l'Université de Bruxelles)

Plantin, Christian. 2005. L'argumentation (Paris : PUF « Que sais-je »)

Rabatel, Alain. 2008. Homo narrans, tome 1 (Limoges : Lambert-Lucas)

Reboul, Olivier. 1991. Introduction à la rhétorique (Paris : PUF)

Robrieux, Jean-Jacques. 1993. Éléments de rhétorique et d'argumentation (Paris : Dunod)

Roulet, Eddy et al. 1985. L'articulation du discours en français contemporain (Berne : Peter Lang)

Todorov, Tzvetan. 1981. Mikhail Bakhtine, le principe dialogique (Paris : Minuit)

Vanderveken, Daniel. 1988. Les actes de discours (Liège : Mardaga)

van Eemeren, Frans \& Rob Grootendorst. 1996. La nouvelle dialectique (Paris : Kimé)

\section{NOTES}

1. Attesté dès 1752 selon le Robert, ce terme a été autrefois employé en France avant de céder la place à son doublet « vote ». Il s'agit actuellement d'un régionalisme, circonscrit à la Suisse, mais également au Québec, pour désigner les consultations démocratiques.

2. Le Conseil fédéral constitue l'organe exécutif de la Confédération suisse. Il est formé de sept membres élus pour un mandat de quatre ans renouvelable. Son rôle est de diriger les affaires 
nationales (défense, budget fédéral...) et la politique étrangère suisse. Au niveau législatif, il s'occupe des procédures de consultations fédérales et de la rédaction des projets de lois.

3. Pour plus de précisions sur la structure et l'organisation de ces brochures, voir dans ce numéro l'article de Corinne Rossari.

4. Ce contenu est réglementé par la loi fédérale du 17 décembre 1976 sur les droits politiques.

5. Notre étude est complémentaire avec celle ci-après de Corinne Rossari qui se concentre sur les aspects énonciatifs de ces brochures.

6. En cela, ces brochures s'avèrent dialogiques au sens très général de Bakhtine : «L'orientation dialogique est un phénomène caractéristique de tout discours. [...] Le discours rencontre le discours d'autrui sur tous les chemins qui mènent vers son objet, et il ne peut pas ne pas entrer avec lui en interaction vive et intense » (in Todorov 1981 : 98).

7. Ces deux dimensions interdiscursive et interlocutive correspondent aux deux facettes du dialogisme théorisé par Moirand (2002). Cependant, en raison du flou extrême de la notion de dialogisme, nous ne l'exploiterons pas dans cet article.

8. Dans le premier cas, le peuple est invité à se prononcer sur des propositions émanant directement du gouvernement fédéral. Dans le second cas, la votation porte sur des propositions faites par un groupe de citoyens en vue de changer la législation existante. Les initiatives populaires existent en Suisse depuis 1848 , date de la constitution instaurant un État fédéral. Actuellement, 100000 signatures au minimum sont requises pour qu'une initiative populaire soit validée et mise en votation.

9. Notamment par des compléments thématisés en tête de phrase : «En Suisse » - «Au niveau fédéral » - « Au niveau cantonal »...

10. Nous rejoignons Grize (1981: 29) qui insiste sur la composante "explicative» de l'argumentation, associée au docere de la tradition rhétorique, avec sa rigueur argumentative.

11. Le rôle du Parlement est néanmoins prépondérant par rapport à celui du Conseil fédéral. C'est en effet le Parlement qui prend officiellement position sur une initiative populaire, le Conseil fédéral devant ensuite assumer et défendre ses recommandations.

12. Des journaux comme Le Temps (du 14-12-2009) ou L'Hebdo (du 18-01-2010) se sont amplement fait l'écho de ces débats. Il en est de même pour la Télévision Suisse Romande qui, le 18 février 2010, a consacré son émission " Temps présent » aux problèmes éthiques posés par la recherche en biologie et en médecine.

13. Roulet et al. (1985: 60) parlent alors de "discours monologique », basé sur une structure d'intervention autour d'un énonciateur principal. Ce type de réalisation s'oppose au « discours dialogique » fondé sur une structure d'échange.

14. Les topoï sont faiblement interactifs dans la mesure où, comme le note Meyer (2008:116), ils constituent un « hors question pour les protagonistes ", n'étant pas sujets à discussion.

15. Selon la terminologie de Plantin (2005).

16. En Suisse, toute initiative populaire doit être pilotée par un comité d'initiative dont la composition varie entre sept et vingt-sept membres.

17. Celui-ci forme une variante de l'argument pragmatique (voir Reboul 1991), à travers laquelle on apprécie un fait en fonction de ses conséquences positives.

18. Perelman et Olbrechts-Tyteca rattachent la communion au registre épidictique de l'éloge qui caractérise le passage cité de la brochure. En faisant un éloge appuyé des bienfaits de l'ONU, le Conseil fédéral s'efforce de créer une empathie entre les valeurs véhiculées par cette institution et celles que le public reconnaît déjà. Le but recherché est que ce dernier opère un transfert métonymique entre une adhésion aux valeurs de l'ONU et une adhésion à l'ONU elle-même.

19. Comme le montrent Desrosières (2000) ou Ogien (2010), l'argumentation par les chiffres est une constante dans le discours politique. Elle présente l'avantage de condenser au moins trois sortes d'arguments : ceux de l'illustration, de la preuve et de l'autorité. 
20. Cet argument proleptique est néanmoins à double tranchant. S'il finalise fictivement le bon aboutissement de la procédure en jeu, il risque de heurter le public en lui donnant l'impression qu'on lui force la main.

21. Voir Aristote (1967 : 1358b-1362b).

22. La possibilité pour le Parlement et le Conseil fédéral de présenter un contre-projet a été créée en 1891 dans le but de transmettre une option plus conciliante qu'un simple refus de l'initiative.

23. "L'initiative répond aux besoins des Suisses, qui veulent plus de sécurité et des frontières plus étanches. [...] Elle règle le problème de l'immigration de masse incontrôlée. »

24. Ces topoï complémentaires entretiennent la relation conventionnelle suivante : l'ordre est préférable au désordre.

25. Nous reprenons ce concept à Rabatel (2008: 266) qui définit la surénonciation comme «l'expression interactionnelle d'un point de vue surplombant ».

26. Suivant la terminologie de Jacques (1985), s'opposant à l'irénisme, la communication agonale qualifie toute attitude sapant l'harmonie interactive dans les rapports sociaux.

27. D’après Maingueneau (1983 : 16), « le "discours-agent" est celui qui cite, c'est-à-dire celui du point de vue duquel s'exerce l'activité polémique ; le "discours-patient", en revanche, est celui qui est intégré et défait ".

28. Pour la sémiotique, les modalités véridictoires déterminent le " jeu de la vérité » (Greimas et Courtés 1979 : 419) entre les dimensions de l'être et du paraître.

29. "On appelle "autophagie" l'incompatibilité d'un principe avec ses conditions d'énonciation, ses conséquences ou ses conditions d'application. Étymologiquement, l'idée "se mange ellemême", fait surgir l'incohérence d'un propos. » (Robrieux 1993 : 111).

30. «L'application du principe de l'État tiers réputé sûr fera baisser [...] le coût total de l'asile, qui se chiffre en milliards de francs.»

\section{RÉSUMÉS}

Lors des votations populaires en Suisse, le Conseil fédéral fait connaître la position gouvernementale à l'aide de brochures envoyées à tous les citoyens. Celles-ci mettent en jeu une corrélation étroite entre argumentation et interaction, tant sur le plan interdiscursif qu'interlocutif. L'objectif de cet article est de montrer, à partir de brochures représentatives, comment une telle corrélation varie fortement selon les stratégies adoptées par le Conseil fédéral. Ainsi, dans les brochures proposant un projet de référendum, on remarque une interaction limitée, en raison de leur argumentation rationnelle et de leur tendance à se fermer sur le point de vue du Conseil fédéral. Par contre, dans les brochures soutenant ou rejetant des initiatives populaires, on observe une montée au premier plan des procédures interactives. Celles-ci peuvent être convergentes ou divergentes, en liaison avec différentes stratégies argumentatives qui vont de la surenchère à la polémique. Au total, ces brochures gouvernementales nous confirment que l'analyse du discours politique gagne à être repensée à l'aide des modèles dits « dialogaux » (Plantin 2005) de l'argumentation.

During popular elections in Switzerland, the Federal Council announces the governmental position by means of brochures that are sent to all citizens. These brochures are characterized by a tight correlation between argumentation and interaction at two levels: the interdiscursive and 
the interlocutive. The aim of this paper is to show, on the basis of representative brochures, how the argumentative strategies used by the Federal Council affect this correlation. In the brochures proposing a referendum project, for instance, we notice a limited interaction due to their rational argumentation and their tendency to focus on the point of view of the Federal Council. However, in the brochures supporting or rejecting the popular initiatives, we observe a rise in interactive procedures. These procedures can be convergent or divergent, and they are associated with different argumentative strategies that go from "overbid" to polemic. All in all, a study of these governmental brochures confirms that the analysis of political discourse is enhanced by the so-called "dialogical" models (Plantin 2005) of argumentation.

INDEX

Keywords : argumentative overbid, interaction, interdiscursive utterance, interlocutive aim, nuanced argumentation, polemic

Mots-clés : argumentation nuancée, énonciation interdiscursive, interaction, polémique, surenchère argumentative, visée interlocutive

\section{AUTEUR}

\section{MARC BONHOMME}

Université de Berne 